\title{
Robot One: A cross-platform and multi-language simulator developed for educational robotics
}

\author{
Alexander Silva Barbosa* Exuperry Barros Costa* \\ Leonardo Rocha Olivi* Afonso da Fonseca Braga* \\ * Electrical Engineering Department, Federal University of Juiz de \\ Fora, Juiz de Fora, Brazil (e-mails: alexander.silva@engenharia.ufjf.br, \\ exuperry.costa@ufjf.edu.br, leonardo.olivi@ufjf.edu.br, \\ afonso.braga@engenharia.ufjf.br ).
}

\begin{abstract}
Despite the rapid development of the robotics' field, overall the equipment's employed are still expensive or even inaccessible, therefore the ground truth and precise tests must be taken in simulation. Although several simulators are available, in most cases these tools are complicated and require a steep learning curve. This paper presents the development of a robotic simulator which is cross-platform, lightweight, language agnostic, able to simulate differential mobile robots. This simulator takes into account environmental and robot variables, such as constructive and random errors, in a simple way of configuring and accessing. Thus the effort is applied in the required task instead of simulation construction, providing a fast and accurate simulator. The simulator follows the cross-platform paradigm and is compatible with several programming languages through a external binding system. Some tests were performed using path planning algorithms to evaluate the simulator and have shown positive results. The main aim of this work is to produce a simulator ready-to-go in educational environments.
\end{abstract}

Keywords: Robotics, Simulator, Mobile Robotics, Educational Robotics,

\section{INTRODUCTION}

In the last decades mobile robotics has shown a remarkable development, being applied in the most diverse areas, like construction, assembly, leisure, agriculture and many other areas (Angelo (2007)). Despite the rapid development of robotics, overall the equipment used is still expensive, which often makes it inaccessible.

When working with robots it is essential that several tests are performed in order to ensure that everything works correctly and in this context simulation tools are extremely valuable because they allow to perform the tests with the robots without the need to use the real robot, which can greatly reduce the possibility of problems that could damage the robot (Carpin et al. (2008)).

Although simulators are extremely important, being used from teaching purposes to exploration of hostile environments or even competitions, often these tools are complicated to use and require a steep learning curve, and sometimes require specific tools for their use, leading to code rewriting.

In this paper is shown a development of a simulator capable of simulating a mobile robot in a three-dimensional environment. In a course of robotics it is imperative that students have contact with real robots as they play a big role in cross-disciplinary learning (Passault et al. (2016)), however as they are not always available, a tool which allows the use of the robot without the need to have a real robot in hand is fundamental so that the theoretical classes can be put into practice.

Several simulators are available for free or paid, but most of these simulators require a precise configuration of several parameters which often makes it impossible to use them quickly. Thus, the simulator from this work has the proposal of being cross-platform, multi-language, easily usable and does not requiring sophisticated configurations, while allowing the simulation of complex environments with sensors generally not available in most simulators. The name of the simulator comes from the phrase "Robots for everyone" which means that the simulator is free, opensource and transparent to the user.

This paper is organized as follows. In Section 2 is presented a description of the proposed methodology, showing the software concepts adopted along with its pros and cons. In Section 3 the software implementation is shown, covering the simulator user interface and the communication API (Application Programming Interface). Section 4 presents some results and comments about the developed simulator and the tests made with it. General conclusions are made in Section 6 along with the future works.

\section{PROPOSED METHODOLOGY}

The proposed methodology is based on the fact that the simulator must run in all three major computing operating systems (Windows, macOS and Linux) and must be easily controlled from any programming language. In order to achieve these statements it was chosen to split 


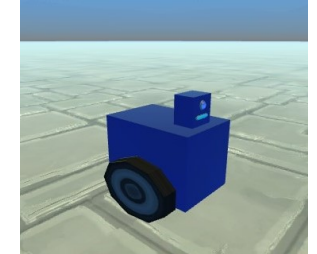

Figure 1. Three-dimensional model of the robot in the simulator.

the simulator in two pieces of software, the $R$-One $U I$ (Robot One User Interface) and the R-One API (Robot One Application Programming Interface). For the $R$-One $U I$ it was decided to use Unity (Technologies (2018)) as the graphic engine due its high flexibility and great set of tools. For the R-One API it was used the C Programming Language and the concepts of Client-Server communication and FFI (Foreign function interface) (Larmuseau and Clarke (2015)).

\subsection{R-One UI}

To develop the R-One UI it was used the Unity 3D Game Engine to create whole simulator interface. Unity was chosen because of its ability to generate binaries for most the current operating systems and its great set of high quality tools, allowing a fast development of the simulator.

Unity projects can be developed using the C\# programming language which due its flexibility and set of libraries allows to do more with less code, simplifying the development of new devices and functionalities.

As the simulator works in a three-dimensional space, the robots and all other objects were modeled based on objects in the real world. In order to allow simulations very close to what happens to a robot in the real world, were defined three main requisites which must be satisfied, being them:

(1) A three-dimensional environment which is able to simulate three-dimensional sensors such as cameras and rangefinders.

(2) Fidelity to the constructive parameters of a differential robot on its kinematic modeling.

(3) Fidelity in the physics simulation of the environment where the robot is placed.

With the requirements defined the simulator objects were modeled. For the robot a generic differential model was modeled along with its sensors, which is shown in Fig. 1.

As the robot have just two wheels to be controlled, its position in the simulation world is calculated considering the velocities of the two wheels, $V_{l}$ (left wheel velocity) and $V_{r}$ (right wheel velocity), the time between two simulation frames $(\Delta t)$, the distance between the two wheels $(L)$ and the robot state in the last simulation frame, $x_{k-1}$ and $y_{k-1}$ for the position and $\theta_{k-1}$ for the orientation. With this variables the position of the robot can defined as:

$$
\left[\begin{array}{l}
x_{k} \\
y_{k} \\
\theta_{k}
\end{array}\right]=\left[\begin{array}{l}
x_{k-1} \\
y_{k-1} \\
\theta_{k-1}
\end{array}\right]+\left[\begin{array}{c}
\left(\frac{V_{r}+V_{l}}{2} \Delta t\right) \cos \left(\theta_{k-1}+\frac{V_{r}-V_{l}}{2 L} \Delta t\right) \\
\left.\frac{V_{r}+V_{l}}{2} \Delta t\right) \\
\frac{V_{r}-V_{l}}{2 L} \Delta t
\end{array}\right]
$$

The position in the vertical axis $(z)$ is not considered in the equations because the robot stays always on the ground.

\subsection{R-One API}

As the $R$-One $U I$ allows the creation of the simulation world, the $R$-One API allows an user made program to interact with the simulation setting and getting parameters, accessing the robot sensors and actuators and performing complex tasks which would not be possible with just a graphical user interface.

For this API were defined a set of requirements in order to maximize the number of people able to use the simulator, removing barriers such as an specific the programming language and a dependence on powerful hardware. There requirements are:

(1) Easy to be programming language agnostic

(2) Ability to be implemented in a real robot

(3) Computationally efficient and lightweight

With the requirements as a base it was chosen to separate the $R$-One API in two pieces that work together, $R$-One Server API and R-One Client API. This separation allows that a real robot implements the $R$-One Server $A P I$ so it can be controlled using the same code used to control the simulator, with the only changing needed being the address of server. At the same time, as the $R$-One Client API just communicates with a server, it can run in another machine like another computer or even a mobile phone. The Fig. 2 shows the architecture of the $R$-One API.

R-One Server API The server part of the API is a piece of software that implements a network server and directly controls the simulator. For the server technology it was chosen to use the TCP (Transmission Control Protocol) (Postel (1981)). This decision was based on the many advantages of the TCP over other protocols like UDP (User Datagram Protocol) (Held (2002)), even considering its large overhead which is the main disadvantage of TCP in this case:

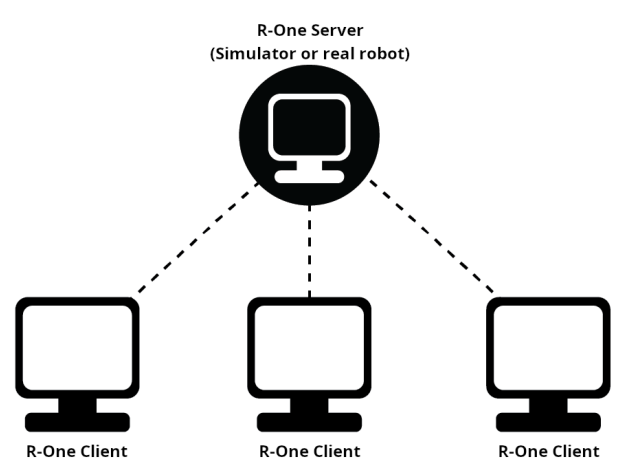

Figure 2. $R$-One API parts communicating in a network. 
(1) Reliability - The protocol can guaranty the package delivery.

(2) Orderly delivery - The protocol utilizes sequence numbers to guaranty the correct reconstruction of a package when it is splitted during the sending process.

(3) Full duplex - Packages can be transferred in both directions at the same time.

Above the network protocol, the server implements a data protocol which allows to control the whole simulation, from the user interface to the constructive parameters of the robot. This data protocol also allows to access the robot sensors and acquire readings.

R-One Client API The role of the client part of the API is to provide an easy way for clients to communicate with the R-One Server API. To be able to communicate with the server the client API implements a TCP client and uses the same data protocol used in the server.

The client API was designed with the FFI concepts, a methodology where functions are defined in a Guest Language which in this case is the $\mathrm{C}$ language and another programming language, also known as Host Language, calls the Guest Language functions through a compatibility layer or bindings.

The $\mathrm{C}$ language was chosen for the FFI interface because almost all programming languages support FFI with C, some examples being: C++, Python, C\#, Java, Matlab, D, Go, Fortran and many others. This feature allows the user to use its most comfortable language, speeding-up the software development.

At the present moment, were implemented bindings for Python and Matlab but as there is the R-One Server API running in the simulator, it is not a must to use the client API, allowing programs to communicate with the server without any API, using just the TCP protocol. This allows the $R$-One API to be compatible with any programming language which can use the TCP protocol.

\subsection{Comparison}

The proposed methodology was compared to the methodologies applied in some of the most popular robotic simulators so that was possible to analyze the pros and cons of both.

Gazebo Gazebo (Koenig and Howard (2004)) is a general robotics simulator developed by the Open Source Robotics Foundation, which allows simulating various robot models and environments. As Gazebo is extremely generalist it does not allow simulations without previous configuration of the robot to be used.

A disadvantage of this simulator is that the control of the robots must be done with the Gazebo API which is a communication and control interface for the simulator using the $\mathrm{C}++$ language, making not possible to natively use another language. An alternative to use the gazebo with other languages is to use ROS (Robot Operating System) (Quigley et al. (2009)), a framework for creating software for robots.
Unlike Gazebo, the proposed simulator can be natively used in several programming languages, however, it is notable that in the current state, mainly due to its maturity, the Gazebo offers a vast amount of features not yet present in the developed simulator.

$V$-Rep V-Rep (Rohmer et al. (2013)) is a robotic simulator based on a distributed architecture where each model or object can be controlled individually through scripts. Since each object can be controlled individually in V-Rep, creating a simulation involves more than simply connecting to the simulator and using the robot as it is necessary to define the joints of the robots in order to obtain their kinematics, requiring a great time setting up the simulation.

In the proposed simulator only the robots are controlled externally, and the control protocol of the simulator offers a separate access to control the robot already totally modeled or each one of its moving parts. Objects other than the robot are not accessible for control, being in charge of the simulator physics engine to take care of them.

Player/Stage Player/Stage (P. Gerkey et al. (2003)) is a pair of a simulator and a network server for robot control. The Player is the software part responsible for creating a TCP server allowing its control through an API, allowing the simulator to not depend on a specific language. The Stage is a simulator used in conjunction with the Player to allow the simulation of a population of mobile robots, objects and sensors.

This simulator is the closest to the proposed simulator, since it uses a network protocol for communication. The difference with this simulator comes from the fact that it is not interested in the physical fidelity of the simulation. In the developed simulator this task is simpler since this is one of the pillars used in the construction of the simulator.

\section{SOFTWARE IMPLEMENTATION}

With the robot (Fig. 1) and its kinematic equations (1) defined, the software development was done modeling the environment for the robot and implementing its kinematics.

\subsection{Sensors}

With this basic implementation, it was modeled the sensors used in the robot. For this robot it was used three sensors, being them: a GPS sensor (Global Positioning System) along with a compass, a LiDAR sensor (Light Detection and Ranging) and a RGB camera.

As in the real world all of these sensor are susceptible to errors in its measures, the simulated sensors must model and implement these errors. With exception of the camera sensor, in all other sensors in the simulation were added Gaussian errors in its measurement. Thus, the user is able to adjust the sensor standard deviation, to emulate a the sensor accuracy as desired.

The GPS sensor in the simulator returns the position of the robot in meters in a plane from the start point of the robot in the simulation, and the compass returns an angle 


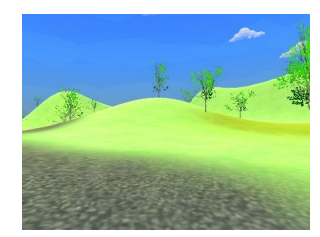

Figure 3. Image captured with the virtual camera.

in radians between the robot and the simulation north direction. Being $X_{\text {real }}$ and $Y_{\text {real }}$ the real coordinates of the robot and $\theta_{\text {real }}$ its real orientation in the simulation, the GPS sensor position combined with the compass orientation can be described as:

$$
\left[\begin{array}{c}
X_{r} \\
Y_{r} \\
\theta_{r}
\end{array}\right]=\left[\begin{array}{c}
X_{\text {real }} \\
Y_{\text {real }} \\
\theta_{\text {real }}
\end{array}\right]+\left[\begin{array}{l}
N\left(0, \sigma_{x}\right) \\
N\left(0, \sigma_{y}\right) \\
N\left(0, \sigma_{\theta}\right)
\end{array}\right]
$$

where $\sigma_{x}$ and $\sigma_{y}$ are the standard deviations for errors in the $X$ and $Y$ measures and $\sigma_{\theta}$ is the standard deviation in the $\theta$ measure.

The LiDAR sensor (Shan and Toth (2018)) is used to measure distances between the robot and another objects using the principles of light reflection. The virtual LiDAR sensor was placed above the robot base with a vision range of 180 degrees, from -90 to 90 and with the 0 degree placed right in front of the robot. The sensor have a resolution of 5 degrees so it can measure 36 distances. This virtual sensor have a measure limit of 8 meters. With the error added to the virtual LiDAR, its measures can be describe as:

$$
\rho=\rho_{\text {real }}+N\left(0, \sigma_{\rho}\right)
$$

where $\rho_{\text {real }}$ is the correct measure of the virtual sensor and $\sigma_{\rho}$ is the standard deviation of the Gaussian error added to the sensor.

The RGB camera added to the robot is based on a CMOS sensor (Complementary Metal Oxide Semiconductor) (Yadid-Pecht and Etienne-Cummings (2007)) which are sensors formed of an array of photosensitive transducers capable of converting the light energy of a point of the image into an electric charge which is then converted to numerical values and now represents a digital image.

The camera in the simulator uses 8 bit converters so each pixel can be represented in three bytes, one for each color channel like most of the real RGB cameras. The maximum resolution of the simulated camera is 320 pixels horizontally by 240 pixels vertically. Fig. 3 shows an image captured with the virtual RGB camera in the simulator.

\subsection{Physics}

Since the simulator must faithfully represent the real world its physical description must be very consistent. Unity 3D contains in its portfolio a wide range of specific functions for physical simulation, from simple mechanics like gravity to the most complex ones such as the collision of threedimensional objects. In this simulator were adopted two physical mechanics to be simulated, the gravitation and the rigid bodies dynamics. Each of these mechanics implementation is described below:
For the gravitation, it was supposed that the simulation world is exactly equals to the earth in dimension and mass. The modeled robot has a height of 0.4 meters so considering just its three-dimensional model its gravitational center is located 0.2 meters above its ground contact point, however with this configuration the robot is extreme unstable on the floor and prone to fall. To fix this the simulation is done with the gravitational center of the robot 0.1 meters above its ground contact point.

The robot is simulated with a mass of $25 \mathrm{~kg}$ so according to the Universal Gravitation equation the robot is attracted to the ground with a force equals to $F \cong 245.341 N$ that is, with an acceleration of $g=-9.81365 \mathrm{~m} / \mathrm{s}^{2}$.

In the simulator is required that when the robot collides with any object the forces involved act as in real world and for this is used the rigid body dynamics (Rao (2006)). Applying this to the robot it is possible to affirm that it can not occupy the same space of another object since the position of each of its particles in relation to the others is constant in time. Considering that during a collision the modules of the forces exchanged by the collision agents are much higher than the modules of the external forces, the system can be considered mechanically isolated.

Using these two facts is possible to calculate the movement of the robot and any object colliding to it using the Conservation of linear momentum equation:

$$
M_{1} V_{1}+M_{2} V_{2}=M_{1} V_{1}^{\prime}+M_{2} V_{2}^{\prime},
$$

where $M_{1}$ and $M_{2}$ are the masses of the two objects colliding, $V_{1}$ and $V_{2}$ are their velocities before the collision and $V_{1}^{\prime}$ and $V_{2}^{\prime}$ are their velocities after the collision.

\subsection{Environment}

In the simulation environment were implemented two working modes: Realistic and Maze. In the realistic mode the robot is placed in an open world scene with four different biomes where is possible to find trees, grass, roads, civil constructions and walls. This mode allows the robot to be used in a place close to what is found in real life.

In the maze mode the robot is placed in a large plane and objects can be added dynamically so it is possible to construct a simulation according to the needs of the user. The maze mode allows the addiction of objects using the $R$-One API and among the objects supported are: Line on the ground, walls, robots, spheres and cubes.

To add the objects is possible to use a text file describing the simulation or an image file where different colors represent types of objects. While texts files offer a simple way to create a simulation, the image files allow the creation of big and complex simulations.

Since the images do not necessarily have the actual size of the simulation map, similar regions must be grouped in the image so that they are summed up to a single point or line. To do this, the Flood Fill algorithm (Burger and Burge (2010)) is used to segment the image into several elements. When the elements are segmented, a thinning morphological operator is applied to the image, so any 


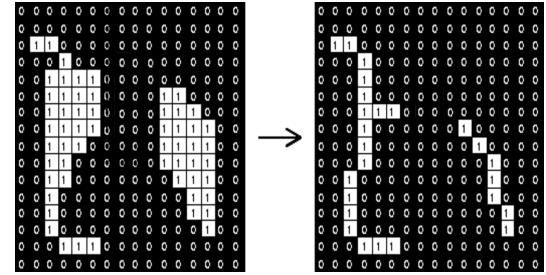

Figure 4. Thinning process of an image.

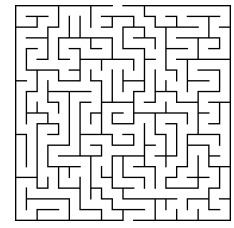

(a) Maze map image.

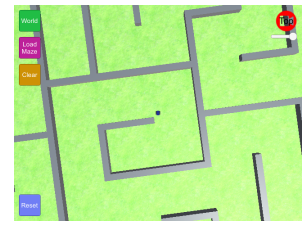

(b) Maze map imported to the simulation.
Figure 5. Maze map.

line or wall that contains more than one pixel in width is reduced so that only lines and walls of one pixel are present in the resulting image. The Fig. 4 shows the thinning in an sample input image while Fig. 5 shows the same operation applied to an maze map in the simulator.

\subsection{R-One API}

The simulator was developed in way that as soon as it starts it also starts an instance of the $R$-One Server API allowing clients to connect to the simulator. When there is no client connected, the simulator listen to keys on the keyboard and on connected joysticks so it allows the user to control some simple things in the simulation without code.

When a client is connected to the $R$-One Server API the simulator stops listing to keys and give the whole control to the client so it can do anything with the simulator. To prevent users for requesting different things to the simulator at the same time, it blocks any new client connection while there is one active connection. As soon as the client disconnects the simulator goes back to the manual mode and starts to accept new client connections.

The $R$-One Server API was designed so that it is always a passive agent in the communication, i.e., it can not control the simulator without a client.

\subsection{Final implementation}

The final simulator implementation is composed of the fully functional $R$-One $A P I$ and $R$-One $U I$, which allows the simulation visualization and its manipulation though a simplified interface. The Fig. 6(a) presents the simulation in the realistic mode in flat land biome. The Fig. 6(b) presents the simulation in the maze mode with a map used for localization in robotic classes, loaded using an image file.

\section{PRACTICAL RESULTS}

Were performed experimental tests to validate the simulator based on its initial purpose. To check if the simulator

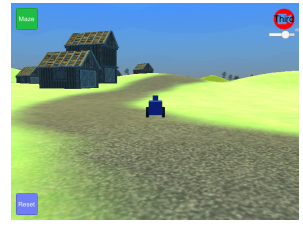

(a) Realistic mode in flat land biome

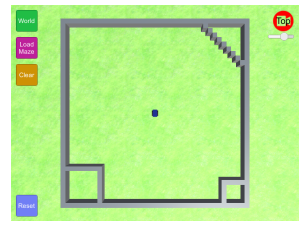
ing a localization map (b) Maze mode simulat-

\section{Figure 6. R-One UI}

was able to being used in class environment, two path planning experiments were chosen to be implemented in the simulator, one covering kinematics using a PID controller (proportional-integral - derivative) and one covering the sensors, using the robot camera to make it follow the road in the realistic mode.

\subsection{PID Controller}

For this test a simple program was implemented using the python language to control the robot using a classic PID, represented by:

$$
u(t)=K_{\rho} e(t)+K_{I} \int_{0}^{t} e(\tau) d \tau+K_{D} \frac{\partial e(t)}{\partial t}
$$

This equation is applied to the angular velocity of the robot, while for the linear velocity a classical $\mathrm{P}$ controller (proportional) is used.

Defining $\rho$ as the distance from the robot to the target point, alpha as the angle between the robot and the target point and $t$ as the simulation time, the equations for the robot control can be described as:

$$
\begin{aligned}
& V_{t}=\min \left(K_{\rho} \rho_{t}, V_{\max }\right) \\
& \omega_{t}=K_{P} \alpha_{t}+K_{I} \sum_{k=0}^{t} \alpha_{k}+K_{D}\left(\alpha_{t}-\alpha_{t-1}\right),
\end{aligned}
$$

where $V$ is the linear velocity, $\omega$ is the angular velocity and $K_{\rho}, K_{P}, K_{I}$ and $K_{D}$ are the controller parameters.

Implementing the python code to apply these equations to the robot and using a sequence of points which describes a star shape, it was possible to see that the robot worked as expected, following the target points in a smooth path. This behaviour can be seen in the Fig. 7(a) and Fig. 7(b).

\subsection{Road follower}

The second test accomplished with the simulator is a simple program which captures the an image with the robot camera and process it to detect the road present in the realistic mode of the simulator. After detecting the road the program must make the robot follow the road.

After capturing the image, which can be done with just one command, the RGB (Red, Green, Blue) space is converted to the HSV (Hue, Saturation, Value). This conversion allows to analyze the color ignoring the saturation and the brightness.

After detecting the mean color value for the road, the program finds the central point of this color in the image, 


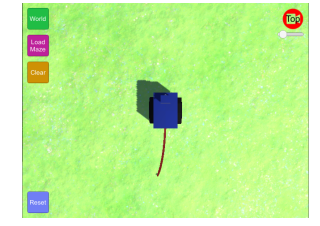

(a) Robot going to the first point.

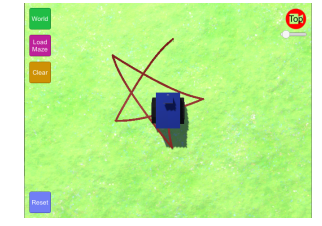

(b) Robot going to the last point.
Figure 7. PID Controller simulation.

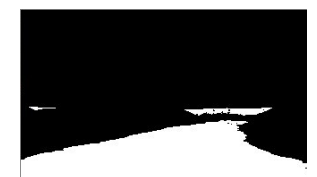

(a) Result of the road (b) Robot following the detection.

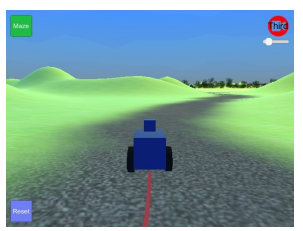

road.
Figure 8. Road follower simulation.

which corresponds to the center of the road. With the central point detected, the angle between the robot and the point is calculated and from this angle is generated an angular velocity. The calculated angular velocity along with a fixed linear velocity is applied to the robot.

The process of detect the road can be seen in Fig. 8(a) and Fig. 8(b) shows the robot effectively following the road. It can be seen that computer vision algorithms can be performed in this lightweight simulated environment successfully.

\section{CLASSROOM EVALUATION}

The Robot One was used during a semester in the Federal University of Juiz de Fora as a first classroom experiment and validation by the students in the Mobile Robotics class. As stated by the class Professor, the simulator has contributed to the teaching of the discipline where there was no complaints from the students and the simulator has worked as expected. During the experiments, some students found some bugs in the software, which were reported and corrected, showing that there was student engagement.

\section{CONCLUSION}

This paper presented a development of a new robotic simulator with the main purpose of being used in robotic classes, allowing the implementation of many robotic tasks in a easy way and using any language preferred by the user.

Practical tests where made to validate the simulator and evaluate its real relevance in the academic environment, which have shown positive results as the classes tasks could be implemented without the effort to create and configure the simulation and instead focus in the task development.

The simulator developed still lacks some features available in another robotic simulators, like the ability to simulate more robots and control non robotic objects, but at the same time it has an smooth learning curve and allows the users to focus less in the simulation and more in solving a given problem.
The Robot One is an always-evolving platform and in order to expand its use cases like the others simulators analyzed, some features can be implemented in the future, being the main: the support to robot manipulators, support for the dynamic addition of robots in the simulator.

Another feature planned for the future is the implementation of an interpreter of some programming language, so that it is possible to develop codes using the simulator itself, enabling simulations with intelligent agents acting in the simulation while the main robot is controlled by the user code. As an application of the R-One Server API is also planned the development of a real robot which implements it, so that the same codes developed for the simulator are usable in this robot.

\section{REFERENCES}

Angelo, J. (2007). Robotics: A Reference Guide to the New Technology. Greenwood Press.

Burger, W. and Burge, M. (2010). Principles of Digital Image Processing: Core Algorithms. Springer London.

Carpin, S., Noda, I., Pagello, E., and Reggiani, M. (2008). Simulation, Modeling, and Programming for Autonomous Robots: First International Conference, SIMPAR 2008 Venice, Italy, November 3-7, 2008. Proceedings. Springer Berlin Heidelberg.

Held, G. (2002). The ABCs of TCP/IP. Taylor \& Francis.

Koenig, N. and Howard, A. (2004). Design and use paradigms for gazebo, an open-source multi-robot simulator. In IEEE/RSJ International Conference on Intelligent Robots and Systems, 2149-2154. Sendai, Japan.

Larmuseau, A. and Clarke, D. (2015). Formalizing a secure foreign function interface. In Software Engineering and Formal Methods, 215-230. Springer.

P. Gerkey, B., Vaughan, R., and Howard, A. (2003). The player/stage project: Tools for multi-robot and distributed sensor systems. Proceedings of the International Conference on Advanced Robotics.

Passault, G., Rouxel, Q., Petit, F., and Ly, O. (2016). Metabot: A low-cost legged robotics platform for education. In 2016 International Conference on Autonomous Robot Systems and Competitions (ICARSC), 283-287.

Postel, J. (1981). Transmission control protocol. Technical report.

Quigley, M., Conley, K., Gerkey, B.P., Faust, J., Foote, T., Leibs, J., Wheeler, R., and Ng, A.Y. (2009). Ros: an open-source robot operating system. In ICRA Workshop on Open Source Software.

Rao, A. (2006). Dynamics of Particles and Rigid Bodies: A Systematic Approach. Cambridge University Press.

Rohmer, E., Singh, S.P.N., and Freese, M. (2013). Vrep: a versatile and scalable robot simulation framework. In Proc. of The International Conference on Intelligent Robots and Systems (IROS).

Shan, J. and Toth, C. (2018). Topographic Laser Ranging and Scanning: Principles and Processing, Second Edition. Taylor \& Francis.

Technologies, U. (2018). Unity 3d. URL https:// unity3d.com.

Yadid-Pecht, O. and Etienne-Cummings, R. (2007). CMOS Imagers: From Phototransduction to Image Processing. Springer US. 